\title{
An Analysis of the BBC Documentary "the Earth" from the Perspective of Audio-visual Language
}

\author{
Qingxue Gao \\ Zaozhuang University \\ Zaozhuang, China 277160
}

\begin{abstract}
As the famous BBC ecological documentary throughout history, "the Earth" performs a scene of wonderful visual feast of the earth by using natural ecological elements to state the story. This paper chooses several pieces of the documentary to analyze, and interprets from the aspects of its film structure, scenes, sound, and montage, so that readers can understand how the film influence the audience by using their own audio-visual language.
\end{abstract}

Keywords-"the Earth"; documentary; environmental protection

\section{INTRODUCTION}

"The Earth" produced by BBC of the UK is a very large eye-catching documentary with the length of about 90 minutes, which starts with stories of three families of species including polar bears, elephants and whales, combining narrative with artistic, and presents the love for beautiful natural environment and worship of the earth in the kinship of animal.

\section{A BRIEF ANALYSIS OF THE STRUCTURE OF DOCUMENTARY "THE EARTH"}

From the perspective of structure of the film, it is divided into three parts. Editing is one of the cores of film production. The editor creates discontinuous, cross-regional, intuitive images with a unique logical thinking form, and the contents form unfolds the overview in the structure of general-dividegeneral, starting from the general overview of the earth and then describing the three species of polar bears, elephants and whales in terms of seasonal changes. The story begins from the polar night and Arctic of the earth and then develops to the south of the earth. When the polar night and aurora of the Antarctic pole appear, the story returns to the north of the earth again. This expression way is like a wanderer who returns home again after long time away from home and feels warm as ever. The end goes back again to the Arctic pole, where snow has melted, and then the film began to tell the story of the polar bear. The film has rich level, with the parallel development of several parallel lines highlighting the theme, which makes the main line clearer and the rhythm more lucid, so that the audiences have deep memory of the beautiful things on the earth. The end and the opening echo each other, and repeated use of scenes is skillful but not bored, which strengthens the consciousness that people need to protect the home of earth.
The structure of these three parts determines the overall framework of the film, so the content starts a specific story narrative in this framework. The film content of each part is narrated respectively from the overall summary to divide narrative, and the picture connection has very good logic, which leave a deep impression to the audience. For example, the design of the opening starts with mysterious music and macro scenes, and briefly summarizes the evolution history from the birth of the earth to the birth of life with intuitive image of pictures.

\section{ANALYSIS OF THE SCENE OF THE DOCUMENTARY "THE EARTH"}

The scene as the smallest unit of the structure of the film carries the transmission of film information with different forms and content. The scene connection and scene conversion of the film are implemented based on conversion of time and space with strong logic. It is unfolded in chronological order, with clear levels of paragraph composition. It mainly adopts chronological narrative, starting with the Arctic Highlands in January which have not seen the sun for more than a month to describe the polar bears foraging; then the scene shifts to the first ray of sun coming out of the horizon in February when interesting stories happens to polar bears and their cubs after winter, which allows the audience to know the living environment of the creatures. This content is full of narrative. The North in March is still covered with ice and snow. The film uses an overlook scene to convert the scene to a vast coniferous forest - a wasteland. The scene turns to the animals in the wasteland with the voiceover, depicting the life of the bobcats with the front and opposite scenes; and then finishes this scene with overlook scene. The image of the rotation of the earth appears in the scene, which directly reflects the changes brought to the coniferous forest by spring, and reflects the important role of the forest for the earth and mankind from the side. Everything in the forests is vibrant in April, and many creatures completed the process of predation and breeding through large-scale migration. In this paragraph, overlook scene and the push and pull and moving of the scene shows the spectacular scenes of animal migration. In addition, the film is good at using the sports scene. For example, in the scene where a wolf prey on the deer separated from the deer herd, the scene takes the moving direction of the wolf and deer as the center, making the image maintain the same moving direction. This part of 
paragraph maintains the same moving direction, i.e., moving from the lower right corner to the top left of the image. The ups and downs of plot deeply cause the audience's mental tension. The operations of shooting and editing that change the direction in the movement need attention. Under the premise of changing the direction of moving objects in the picture, keeping the fluent narrative is not a simple operation that can be completed in an action. In the picture, if the direction of moving objects are the same, the contradictions and conflicts of moving objects are visually manifested in two variable motions with consistent directions, and the viewer can confirm the direction of the moving objects in two scenes by the recognition of the moving object, and thus infer their movement is what kind of movement logically. The scene of wolf chasing deer in the film is performed very well. The editor takes the escape route of the deer as the center of screen, followed by the use of moving scene. Such a flexible content of scene drives the jump of viewers' thinking.

The large number of time-lapse photography scenes in the film is one of the highlights. Analyzing from the visual language and the law of the scene, the more important narrative content is, the more medium shots and close shots should be used in shooting scales of the image; otherwise, long shot and panorama should be used. 1After the scene of wolf chasing deer, the scene uses the method of time-lapse photography, and switches the scene to farther southern area coordinating with ingenious scene design. Under the conditions of polar day, everything is exuberant. Proximity film editing and time-lapse shooting including long shot, panorama and close shot are used to successively introduce the lives of several types of animals, showing the beauty of changes in four seasons of one year with rapid changes in time. With the help of the records of these beautiful scenes, the film reflects to the audience that human's transformation of the nature makes the circulation of four seasons more and more unreliable from one side.

As time goes by, May comes. Spring can no longer be found in tropic in May, since twelve hours sunshine everyday makes the jungle grow freely as well as nourishes a lot of lives. The scene converts to the growth a group of mushroom plant, apes picking figs, courting of bird of paradise that are full of fun with a close shot. In the shooting of the birds of paradise, the film uses a lot of close shots and close-up shots to show the type and life of them, which is lively and interesting.

The opening of each paragraph of the film will generally show the majestic panorama, and then it is detailed into the animals and plants in close shot. This subtle connection of pictures enhances the narrative of the scene and makes the picture look soft without jump. Next, the scene depicts the grand spectacle of expansion of the desert on the earth, which is contradict to and suitable for the former scenes with compatible environment. This contrast reflects the living conditions of creatures in the desert is severe than ever before. The connecting scene draws forth the Kalahari Desert

Qi Jianming, Xia Pingting. Audio-visual language in animation. Shenyang: Liaoning Fine Arts Publishing House, 2011, 12, pp.26 in South Africa in June that is in dry season. The elephants begin the journey to find water and food, so all the elephants head to Okavango together. In this paragraph, the close-up scene of the elephant's legs leads to the next picture of the elephant group. It can be seen from the dry and hot desert environment and the sand in the scene that the journey of elephant group is not easy. For this purpose, in the latter scene a pair of straggling elephant mother and child after a dust storm is depicted in detail. In this piece of film, the bison herd also joins the journey of finding water. The scene is shot from the front to the overhead, showing the love and desire for water of lives. Flat green grassland also demonstrates the lifecycle over and over again. The whole scene is edited smoothly and understandably, with the perfect scene control making the story of the film intertwined and interlocked.

When the film tells the story of seven humpbacks, it uses mobile scheduling to make the scene move with the movement of the whale in the picture, making full use of dynamic performance of the scene to let the audience's attention focus on the movement process of the whale. Beautiful blue ocean and whale perfect fuse together, so that the audiences once again feel the shock brought by everything in the nature.

\section{A BRIEF ANALYSIS OF THE SOUND OF DOCUMENTARY "THE EARTH"}

The combination of sound and picture jointly forms the artistic thinking way of the film. Sound can not only render the environment, but also participate in the narrative. Each piece of music in the film is combined with the picture, of with the changes are harmony and wonderful. The beautiful symphony and scenes are intertwined in the film, with the combination of sound and picture promoting the narrative. Wonderful narration and perfect tone quickly bring the audience into the beautiful natural environment. In the scene of wolf chasing deer in the first half of the film and scene of polar bear capturing the walrus in the second half, the music rises and falls with the intense chasing atmosphere, pushing this scene to the climax.

Wonderful picture joined with the timely sound is enough to express the full meaning of the film and interpret the mental state of the creator. In the film there is relaxed and lively music sometimes. For example, when the bears play in the sun after the thaw of snow and ice, birds of paradise fly in the vast forest, and monkey and elephant cross the river for fun, relaxed music is played in these parts. This not only shows the interesting side of nature, but also expresses the creator's love for the natural world.

In addition, the sound has the coherent effect of connecting the scenes. When many paragraphs in the film come to the end, the music will connect the two scenes, serving as the transition. At the 55th minutes in the film, the scene shows demoiselle crane flying over the Himalayas for winter. The background music and sound effects make the scene cover the clouds and then slowly dissolve, transiting to the next scene of a river. The next scene has been showing mountains and waterfalls, with distant symphony 
accompanying by spectacular pictures, and the quiet and beautiful atmosphere arises spontaneously.

\section{SEVERAL MONTAGE EXPRESSING Forms OF DOCUMENTARY "THE EARTH"}

The creation of this film uses a large number of montage expressing forms. This paper analyzes several points of the film.

\section{A. The Use of Narrative Montage}

The film is good at using narrative montage to edit together the plots at same time in different regions alternately. For example, in the journey of elephant finding water, it arranges the scene of buffalo finding water alternatively. Each plot depends on each other, completing the narrative together.

\section{B. The Use of Reproduce Montage}

In the process of wolf pursuing the deer, the film uses the reproduce montage expressing form, constantly showing the scene of wolf pursuing the deer of which the content is consistent with nature, which highlight the sense of urgency step by step. It is very easy for such effect to infect the audience.

\section{The Use of Lyrical Montage}

The use of lyrical montage is very common in this documentary. This is a kind of thought and emotion that guarantees the coherence of narrative and description and performs beyond the story. ${ }^{2}$ This montage expressing form is very widely used in the whole film. For instance, in the emergence of polar bears and elephants and the introduction of the tropic in May, lyrical montage is applied.

From 56th minutes, the film begin to introduce the impact brought by the melting of snow and ice due to the sunshine The scene uses overlooking shoot, rotating scene, and moving shot and so on to show the spectacular waterfall. Shooting the impetuous waterfall until the emergence of rainbow from multiple angles can make the audience look forward the content of next paragraph. The emergence of rainbow brings new power to this piece, indicating the new vitality and good news. The next scene is the emergence of the bison herd that finally reaches the end of a long journey.

\section{The Use of Metaphoric Montage}

The use of metaphoric montage in the film is produced by contrasting the large scene. For example, the contrast between the beautiful environment and the bad weather implicitly and vividly shows the creator's creative intention, which will lead the audience to think and raise their environmental awareness.

The end of the film once again tells the story of the polar bear. With the acceleration of melting of iceberg caused by global warming, the male polar bear find a place to rest after

\footnotetext{
2 Qi Jianming, Xia Pingting. Audio-visual language in animation. Shenyang: Liaoning Fine Arts Publishing House, 2011, 12, pp.108
}

seeking on the sea for several days, but the hungry polar bear did not get the opportunity of predation. At sunset, it lies on the ground tired and hungry. The scene turn to the new picture, where two polar bear cubs appear again, and they grow sturdily after leaving the mother bear. The two cubs are playing in the snow and having fun in sea with floating ice. As the commentary says, the polar bear has become the current situation of the Earth and a symbol of all the animals that endeavor to survive with us together. These offspring are the proof of life adaptability. This sentence is memorable. At the end of the scene, a polar bear swims in the sea with ice floating around, which again makes the audience recognize the importance of protecting the earth's environment. It is protecting the human ourselves to cherish the natural environment, do not let it suffer from the strong destruction of modernization, and coordinate the coexistence with other creatures.

\section{CONCLUSION}

"The Earth" is a very good documentary. This film is a beautiful natural feast for the audience as well as a very good textbook on shooting and editing for the film lovers. The film not only allows the audience to learn the professional shooting and editing knowledge, study the excellent audiovisual language, but also can encourage the audience to reexamine their own environmental awareness, which is worth the profound reflection of every audience.

\section{REFERENCES}

[1] Qi Jianming, Xia Pingting. Audio-visual language in animation. Shenyang: Liaoning Fine Arts Publishing House, 2011, 12

[2] Li Haiying. Audio-visual language in animation. Nanjing: Nanjing University Press, 2010 